\title{
Weak lensing at the limit of the sky background noise
}

\author{
Yannick Mellier ${ }^{1,2}$ and Bernard Fort ${ }^{2}$ \\ 1 Institut d'Astrophysique de Paris CNRS, 98 bis Boulevard Arago, 75014 Paris, France \\ 2 Observatoire de Paris, DEMIRM, 61 Avenue de l'Observatoire, 75014 Paris, France
}

\begin{abstract}
Recent weak lensing observations have pushed the use of 4 meter-class telescopes to the limits of their capabilities with exposure times exceeding several hours. The leading idea is that the surface density of faint galaxies up to very faint magnitude $(B>28-30)$ raises continuously thus potentially offering a dense template of distant sources whose intensity contrast is at the sky noise level. In complement to the Peter Schneider's presentation on dark matter search from weak lensing (this conference), we review some of these recent advances in weak lensing analysis based on this extreme faint population of galaxies in order to explore:

1. the dark matter distribution on large scales,

2. the redshift ditribution of lensed sources at very large distance,

3. and eventually the values of cosmological parameters.

For each observational topic we will briefly discuss these new methods as compare to more classical lensing studies as well as the possible VLT scientific impact in the domain.
\end{abstract}

\section{Cosmology with gravitational lenses}

Gravitational lenses effects on distant galaxies can potentially probe either the dark matter distribution from low mass compact objects to large-scale structures, or the angular distances of sources that depend on their redshift distribution and to a smaller extend on the cosmological parameters. This has motivated a lot of observational efforts devoted to large multiple arcs and arclets in rich clusters of galaxies (see Fort \& Mellier 1994 and Narayan \& Bartlemann 1996 for reviews). Indeed, we still expect important results from the strong lensing regime with the HST and the VLTs since recent improvements in instrumentation or data analysis provide unprecedented observational capabilities with these facilities. But except in the core of compact clusters of galaxies, arc(let)s are relatively rare events which cannot neither easily represent a large and fair sample of faint distant galaxies at large redshifts (luminosity bias) nor probe the mass distribution outside high condensation of masses. In this domain the greatest hope could be that HST observe an exceptional event with several multiple images systems at different redshifts that will allow a unique test on the geometry of the Universe.

So, after the pioneer analysis of Tyson et al (1990) on the shear field around A1689 and the extensive work of theoreticians (see Schneider, this conference), it appears clear that weak lensing can in principle be observed everywhere in the 
sky if we have very deep CCD images of the galaxy population at large redshift: the densest the population of background galaxies, the highest the visibility of the shear effect (better angular resolution and/or higher signal to noise ratio). Simultaneously crucial questions raised to observers: how deep is it possible to go in order to detect a large number of sources that can be statistically used to measure a coherent lensing signal on a given sky aperture with the highest signal-to-noise ratio? Is it actually possible to correct all the atmospheric and instrumental distortions of astronomical images whose amplitudes can be 5 to 10 times larger than the predicted amplitude of the gravitational lensing signal?

A technical answer to the second question is not trivial and far beyond the scope of this paper because all the ground based telescopes are affected by seeing effect and have been so far constructed without such an ultimate image quality in mind. Without a strong and dedicated effort on image correction of non axisymmetric geometrical Point Spread Function, the measurement of weak shear below a few percent may be only possible with the HST on a few limited field of view for a while. This important remark has to be kept in mind all along the reading of this paper because we implicitly suppose that the problem has been solved.

For the first question, we know that for long exposure time with excellent seeing $^{1}$, the photometry of the faintest galaxies is limited to about $B=26.5$ or $I=25$. Beyond these magnitudes, the sources are still there but hidden in the photon noise of the sky background. The good linearity of CCD gives in fact a unique opportunity to detect this underground population and to measure their number density or some global coherent geometrical feature, like the weak shear induced by the deviation of light by condensation of mass. In the following we show first how the pixel to pixel autocorrelation function of the sky background reveals the existence of such a large population of extremely faint galaxies and how it is possible to use them to map the shear with an unprecedented signal to noise ratio. Then we discuss the possibility claimed by Fort et al (1996a) to detect the possible positions of sources down to $0.5 \sigma$ of the sky level $(B=28)$ and to use the number counts to study the magnification bias effect around cluster of galaxies. Such an approach open a new way to explore the redshift distribution of galaxies to limiting magnitudes far beyond the other methods (spectroscopy and lensing inversion), and to search for new constraints on the value of the cosmological constant.

In this review, we focus on these recent developements which use faint sources at the noise level. Though still in their infancy, they will demonstrate their full scientific interest when used with the outstanding capabilities of the VLTs.

\section{The autocorrelation function of pixels}

All standard methods which are used to determine the projected mass density from a lensing inversion of the shear map proceed in the same basic way. Very

\footnotetext{
${ }^{1}$ The meaning of this is not clearly defined. Let say that it corresponds basically to 3-5 hours on a 4 meter telescope with a median seeing below 0.7 arcsec.
} 
faint objects are detected down to a threshold limit that strongly depends on the seeing. Then, the center, shape, size and magnitude of every sources are calculated and averaged within a given solid angle (scanning aperture on the sky) that defines the angular resolution of the shear map. The averaged ellipicity is finally linked to the potential and projected mass distribution on the sky. Though pratical and easy to implement, the method depends on the detection threshold, the convolution mask used for the measurements and the local statistical properties of the noise (Bonnet \& Mellier 1995, Kaiser et al. 1995). Several critical issues of the method, related to the identification and delimitation of individual objects have led Van Waerbeke et al. (1996) to consider faint sources down to the noise level as a global density field and to measure the weak lensing effects from the analysis of the autocorrelation function of pixels (ACF) in CCD images. The concept is simple and can be easily understood: the Fourier Transform of an elliptical distribution of light is a conjugate elliptical distribution of density in the $u, v$ plane with the same ellipticity (rotated by $\pi / 2$ ). Thus the ACF on the angular scale of faint distant galaxies is a new mathematical object that sum up all the $u, v$ sources down to the noise and immediately reveals the shear of all the sources in the scanning aperture. An important point is that ACF avoids measurement of centroids and shape parameters of individual galaxies which considerably reduces the uncertainties coming from sources of errors on the geometry of small and noisy objects.

Following Van Waerbeke et al. (1996) and Van Waerbeke \& Mellier (1996) we can express the relation between the surface brightness, $I(\boldsymbol{\theta})$, in the image plane at the position $\boldsymbol{\theta}$ and the surface brightness in the source plane $I^{(s)}$ by

$$
I(\boldsymbol{\theta})=I^{(s)}(\mathcal{A} \boldsymbol{\theta}),
$$

which can be extended to the ACF (e.g. the two-point autocorrelation function of the light distribution in a given area),

$$
\xi(\boldsymbol{\theta})=\xi^{(s)}(\mathcal{A} \boldsymbol{\theta}),
$$

and the thin lens equation can be re-written as

$$
\xi(\boldsymbol{\theta})=\xi^{(s)}(\theta)-\theta \partial_{\theta} \xi^{(s)}(\theta)[1-\mathcal{A}] .
$$

It proves that the local ACF behaves like a new object. In the image plane $\xi(\boldsymbol{\theta})$ can be understood as the sum of an isotropic unlensed term, $\xi^{(s)}(\theta)$, an isotropic lens term which depends on $\kappa$, and an anisotropic term which depends on $\gamma_{i}$.

The weak lensing information is now given by the shape matrix $\mathcal{M}$ of the $\mathrm{ACF}$,

$$
\mathcal{M}_{i j}=\frac{\int \mathrm{d}^{2} \theta \xi(\boldsymbol{\theta}) \theta_{i} \theta_{j}}{\int \mathrm{d}^{2} \theta \xi(\boldsymbol{\theta})} .
$$

and the shape matrix in the image plane is simply related to the shape matrix in the source plane $\mathcal{M}^{(s)}$ by $\mathcal{M}_{i j}=\mathcal{A}_{i k}^{-1} \mathcal{A}_{j l}^{-1} \mathcal{M}_{k l}^{(s)}$. If the galaxies are isotropically distributed in the source plane, $\xi^{(s)}$ is isotropic, and $\mathcal{M}_{i j}^{(s)}=M \delta_{i j}$, where $\delta_{i j}$ is 


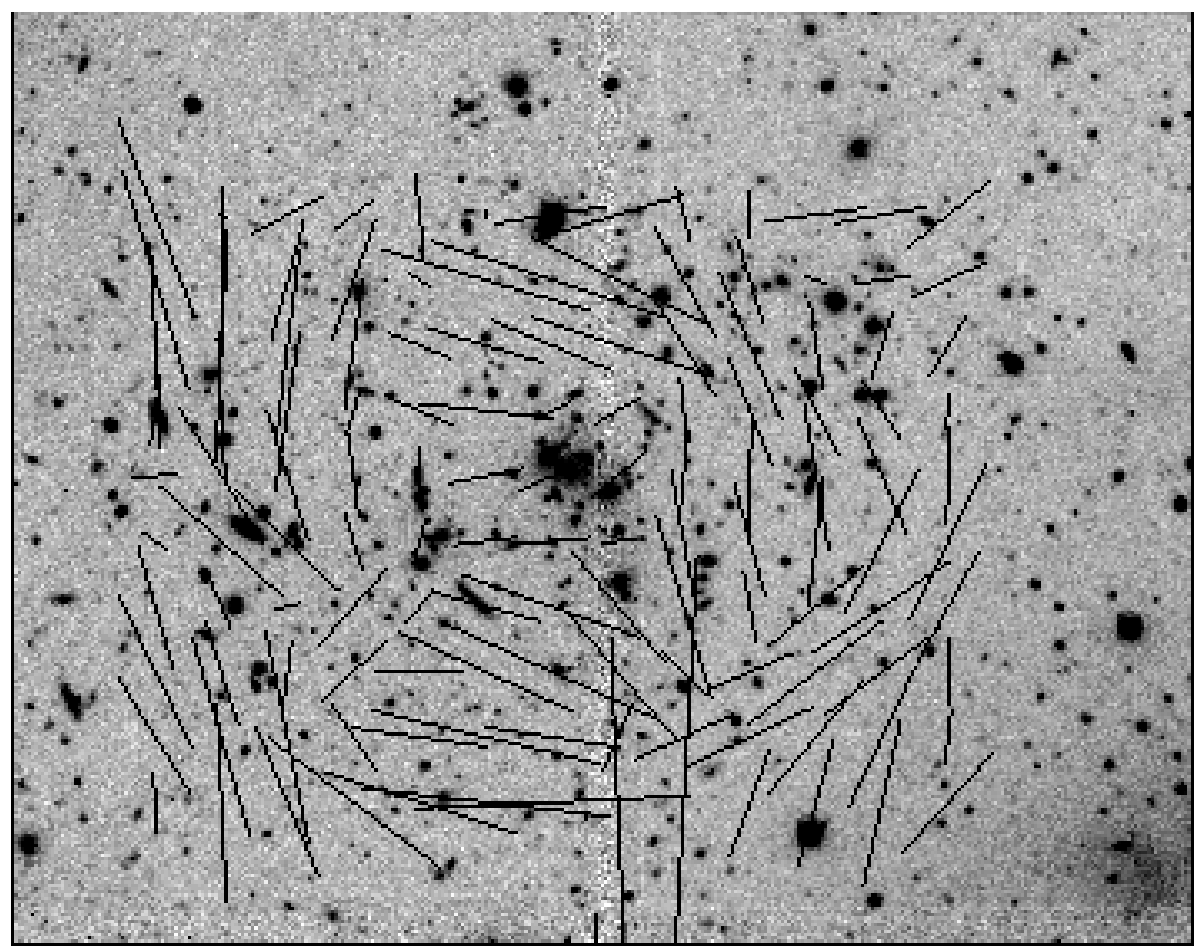

Fig. 1. Shear map around Cl0024 from the ACF method obtained from a mosaic of two CCD images. The map can be compared with the first one obtained by Bonnet et al. (1993). The resolution is better and in particular the innermost pattern shows now that the central mass distribution Cl0024 is bimodal.

the identity matrix. Using the expression of the amplification matrix $\mathcal{A}$ we can formally write $\mathcal{M}$ as follows:

$$
\mathcal{M}=\frac{M\left(a+|g|^{2}\right)}{(1-\kappa)^{2}\left(1-|g|^{2}\right)}\left(\begin{array}{cc}
1+\delta_{1} & \delta_{2} \\
\delta_{2} & 1-\delta_{1}
\end{array}\right) .
$$

Finally, the observable quantities (distortion $\delta_{i}$ and magnification $\mu$ ) are given in terms of the components of the ACF shape matrix,

$$
\delta_{1}=\frac{\mathcal{M}_{11}-\mathcal{M}_{22}}{\operatorname{tr}(\mathcal{M})} ; \quad \delta_{2}=\frac{2 \mathcal{M}_{12}}{\operatorname{tr}(\mathcal{M})} ; \quad \mu=\sqrt{\frac{\operatorname{det}(\mathcal{M})}{M}},
$$

where $\operatorname{tr}(\mathcal{M})$ is the trace of $\mathcal{M}$ and $\operatorname{det}(\mathcal{M})$ is the determinant of $\mathcal{M}$.

As for classical methods, we see that the distortion is available from a direct measurement in the image plane while the magnification measurement requires to know the value of $M$ which is related to the light distribution in the source plane or in any unlensed reference plane. The main advantage of the ACF method is that it provides a new way to measure $\delta_{i}$ and $\mu$ which does not depend upon 
the geometry of the scanning aperture on the CCD image. Furthermore, its signal to noise ratio is proportional to the number density $n$ of background galaxies: $S / N \propto n$ instead of $S / N \propto \sqrt{n}$ for the standard methods (see figure 1).

A full description of the practical implementation of the ACF and first results are given in Van Waerbeke et al. (1996), Van Waerbeke \& Mellier (1996) and Mellier et al. (1996). Clearly, the ACF is potentially the most powerful and promising technique to map very weak shears as those already detected at the outer periphery of clusters, those around bright quasars that seems magnifed by (unseen) condensations of mass (Fort et al. 1996b) or those predicted by large scale structures.

As the signal to noise of the ACF increases as the galaxy number density, the method will be very attractive on deep VLT images with good image qualities (seeing < 0.7 "). FORS should be a unique instrument for some of these programmes.

\section{The distance of background sources with $B>25$}

\subsection{Present status of the $\operatorname{arc}($ et)s redshift distribution}

Spectroscopic redshifts of luminous giant arcs allow to calculate the angular distances $D_{\mathrm{d}}, D_{\mathrm{ds}}$ and $D_{\mathrm{s}}$ (see Eq. (1) of Schneider in this proceedings) and to get the absolute scaling of the lens potential. If arclets are also observed in regions where the potential are properly probed by the modelling of giant arcs it is then possible to infer the most probable redshift of each individual arclet from their position and their ellipticity. The method has been successfully developped by Kneib et al. (1994) using some cluster lenses as very low resolution gravitational spectrograph. The most probable redshift for each arclet is obtained by assuming it corresponds to the source plane where the distortion is minimum. Potentially, it allows to provide redshift $(\delta z \pm 0.1)$ of equivalent un-magnified galaxies down to $B=27$ if the lens modelling is reliable and the shape of each arclet is measurable with a good accuracy. Recently, Kneib et al. (1996) used this technique on deep HST images of A2218 and show that, as for A370 (Kneib et al. 1994), the colour-redshift diagram seems consistent with the deepest redshift surveys. However, because it is a probabilistic approach, its efficiency must be also checked from spectroscopy. This long term programme started one year ago and spectroscopic surveys of the "brightest" arclets in A2218, A2390 and others are underway. Ebbels et al. (1996) and Bézecourt \& Soucail (1996) purposely selected arclets showing bright spots of stars forming regions on HST images in order to detect an emission line and get secure redshift (see figure 2). The rather good agreement between spectroscopic redshifts and lensing inversions demonstrate that gravitational redshift are reliable. However, the observations require a large amount of nights on 4 meter telescopes and cannot be extended to the faintest arclets.

The spectroscopic observation of arc(et)s for the strong lensing modelling, for future cosmological tests, as well as for the study of the distribution and 

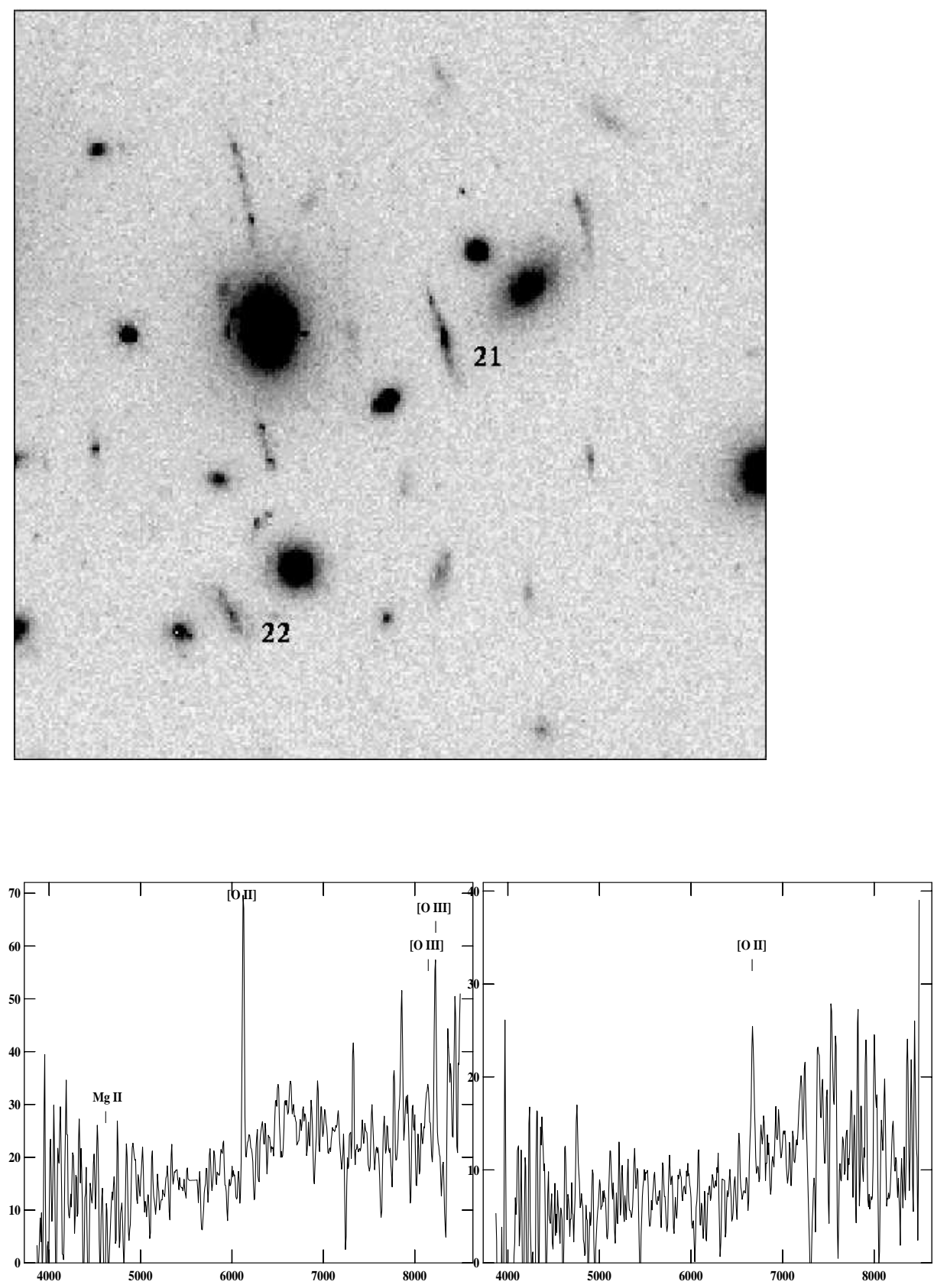

Fig. 2. Spectroscopy of faint arclets in A2390. The top panel is a deep HST image of its central region. The arclets are clearly visible with some of them showing image multiplicity with image parity changes. The arclets 21 and 22 have been observed by Bézecourt and Soucail (1996) with the MOS multiobject spectrograph at CFHT. As expected, they show the $[\mathrm{OII}] \lambda 3727$ emission line from which the redshifts are easily measured $(z=0.643$ and $z=0.790$ respectively. Courtesy J. Bézecourt). 
evolution of faint distant galaxies will be continued with the VLT because one can reasonnably go at least 0.5 magnitude beyond 4 meter telescopes. In fact, one may be able to go a bit deeper by using FORS with the Va-et-Vient spectroscopic mode proposed by Cuillandre et al. (1994). This technique works well in low resolution spectroscopy in particular to derive the spectral energy distribution of faint distant galaxies without emission lines or QSOs with broad emision lines (for galaxies with thin emission line higher spectral resolution may be more suited). According to the gain estimated by Cuillandre et al., the flat field residuals are removed with a much better accuracy than in standard spectroscopic observations and it is possible to go 1 magnitude deeper. In that case, FORS with the Va-et-Vient spectroscopic mode could observe arclets as faint as 26.5 which corresponds to 20-30 arclets per cluster in three hours.

Since the lensing magnification is large, redshift surveys of arc(let)s could probe the redshift distribution of galaxies with $B>24$. But unfortunately, the galaxy sample is biased in three ways. First, only arclets with star forming emisson lines are selected. Second, sky features and redshift effect conspire to offer peculiar windows of redshift visibility. Finally, as it is discussed in the next section, the magnification bias also favours observations of blue galaxies rather than red. So, at present the spectroscopy of arclets is crucial for the lens modelling but the redshift distributions obtained from these methods are still questionnable.

\subsection{Probing the redshift of sources up to $B=28$ with the magnification bias}

Broadhurst (1995) first demonstrated that the radial surface density of background galaxies around a cluster lens like Abell 1689 varies according to the predicted magnification bias of the lens and can be used to measure $\kappa$ directly and to map the projected mass density of the cluster. More attractive, this (anti) magnification bias effect provides a good way to break the intrinsic degeneracy of the inversion methods based on gravitational weak shears: an additionnal plan of constant mass density on the line of sight does not change the shear pattern but just the convergence of light beams.

The radial behavior of the so-called Broadhurst's effect results from the competition between the gravitational magnification that increases the detection of individual objects above the limit of detection and the deviation of light beam that decreases the apparent number counts. Therefore the amplitude of the magnification bias depends explicitly on the slope of the galaxy counts as a function of magnitude and on the magnification factor of the lens:

$$
N(<m, r)=N_{0}(<m) \mu(r)^{2.5 \gamma-1},
$$

where $\mu(r)$ is the magnification factor of the lens, $N_{0}(<m)$ the intrinsic number density in a nearby empty field and $\gamma$ is the intrinsic count slope:

$$
\gamma=\frac{d \log N(<m)}{d m} \text {. }
$$


The radial magnification bias $N(<m, r)$ shows up only when the slope $\gamma$ is different from the value 0.4 ; otherwise, the increasing number of magnified sources is exactly cancelled by the apparent field dilatation and there is no effect on $N(<m, r)$. As noticed by Broadhurst, a radial amplification bias cannot be observed for $B(<26)$ since the slope is almost this critical value (Tyson 1988, Shanks 1996) but it can be detected in the R or I bands when the slopes are close to 0.3 (Smail et al. 1995).

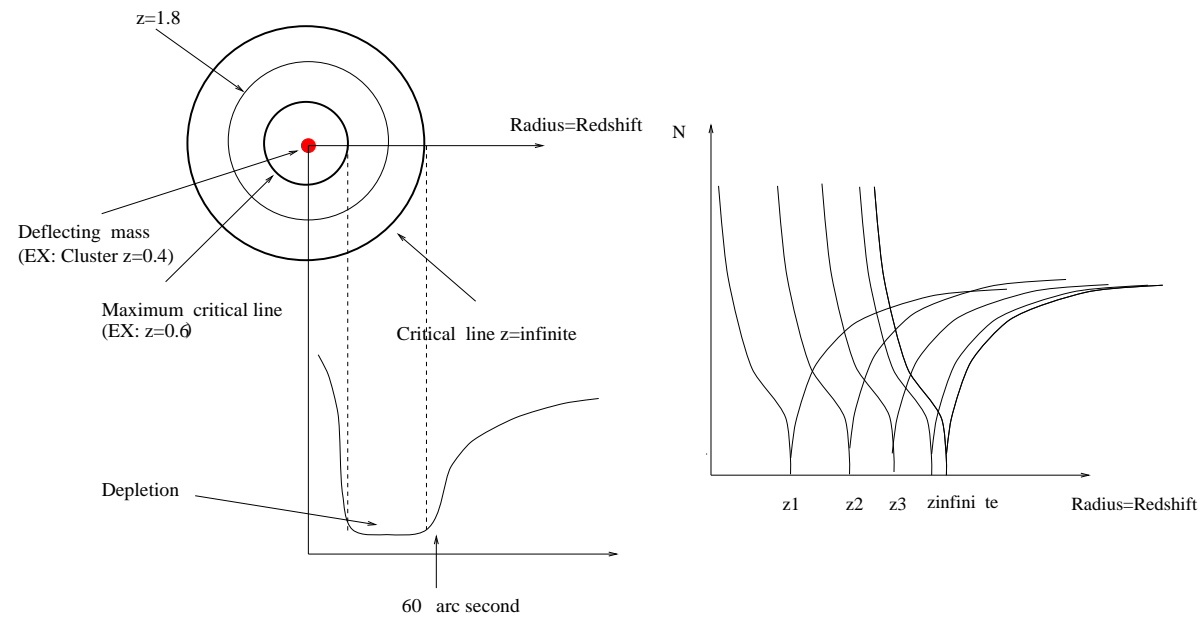

Fig. 3. Measurement of redshifts from depletion curves. The left panel shows the depletion by a singular isothermal sphere as it would be observed on the sky (top left) and the radial projected density of galaxies (bottom left). For a single source redshift, when the lens is perfectly known, the minimum of a depletion curve is sharp and its radial position is formally equivalent to a redshift (for example the position $z 1$ of the right panel). The radial position of the miminum increases with the redshift of sources but the depletion curves tighten and converge towards the curve corresponding to sources at infinity. In a realistic case, the redshift distribution is broad and the individual curves must be added. The bottom left panel shows the depletion as it would be observed: instead of the single peaked depletion we expect a more pronounced minimum between two radii (i.e. two redshifts ) whose angular positions depend on the cosmological constant for high redshift sources. Thus, if the mass distribution of the lens is well known as in the rich lensing cluster Cl0024+1654, the distribution of sources and $\lambda$ can be inferred from the shape of the depletion curve.

When $\gamma<0.3$ a sharp decrease of the number of galaxies is expected in regions of strong magnification close to the critical radius of the lens corresponding to the redshift of the background sources. Since the critical radius increases with redshift, every population of galaxies at different redshifts will display a distinct peaked annular depletion at its own critical line. It can result a shallower depletion between the smallest and the largest critical line which depends on the redshift distribution of the galaxies. (Figure 3 and 4 ). In short, the lens gives us 
a new way to sort out different class of galaxies versus their redshift distribution. It was first used by Fort et al (1996a) with the cluster Cl0024+1654 to study the faint distant galaxies population in the extreme range of magnitude $B=26.5-28$ and $I=25-26.5$ after a detection of the sources in the sky background noise. For this selected bins of magnitude they found on their CFHT blank fields that the counts slope was near 0.2, well suited for the study of the Broadhurst's effect. After analysis of the shape of the depletion curve (figure 4), $60 \% \pm 10 \%$ of the $B$-selected galaxies were found between $z=0.9$ and $z=1.1$ while most of the remaining $40 \%$ galaxies appears to be broadly distributed around a redshift of $z=3$. The $I$ selected population present a similar distribution with two maxima, but spread up to a larger redshift range with about $20 \%$ above $z>4$. In fact, many of the $I$ selected galaxies were not detected in $B$ as if their Lyman $\alpha$ discontinuity has already crossed the $B$ filter band (redshift range $z>3.5$ ).

The main characteristic of this observation is the long exposure time with an excellent seeing condition. Otherwise the detection of such faint objects with an accuracy almost comparable to the HDF would be impossible. In that sense, it is quite clear that such observations are perfectly suited for FORS during the best seeing period on Paranal. So far it seems that the magnification bias is probably a good technique to study the abundance of galaxies at very large distance. Just one limitation comes from the fact that the critical lines of a gravitational lens tend to rapidly merge with the critical line at infinity for a redshift larger than 3. So we can just estimate the relative population of faint galaxies above this redshift. Conversely, this limitation was in turn used by Fort et al (1996a) for a first attempt of a direct measurement of the value of the cosmological constant.

\section{Measuring the cosmological constant from depletion curves}

The qualitative profile of the depletion curve in a direction perpendicular to the critical line is given on figure 3 . When a distribution of detectable galaxies spread up to large redshifts all the most distant ones beyond $z=3$ will form their sharp depletion at almost the same radial distance that, for simplicity, we identify here to $r c_{\infty}$. The most abundant the very distant galaxies are, the stronger is the rising discontinuity at the end of the depletion (figure 4). Basically, Fort et al (1996a) have used the modelling of the lens inferred from the giant arc and the location of the last critical line $R_{I}$ detected in the $I$ bandpass to implement a cosmological test on the value of the cosmological constant. For a flat universe they found $r c_{\infty}$ at a too large distance from the center of the cluster for a non zero cosmological constant ${ }^{2}$. With a thin lens hypothesis and despite large uncertainties their observations seems to favour an $\Omega_{\Lambda}$-dominated flat universe with a cosmological constant ranging from 0.6 to 0.9 . On the other hand, statistics of gravitational lens events on QSOs seems to rule out models

\footnotetext{
2 except if there is an additional, and so far unseen, deflector hidden behind Cl0024+1654.
} 

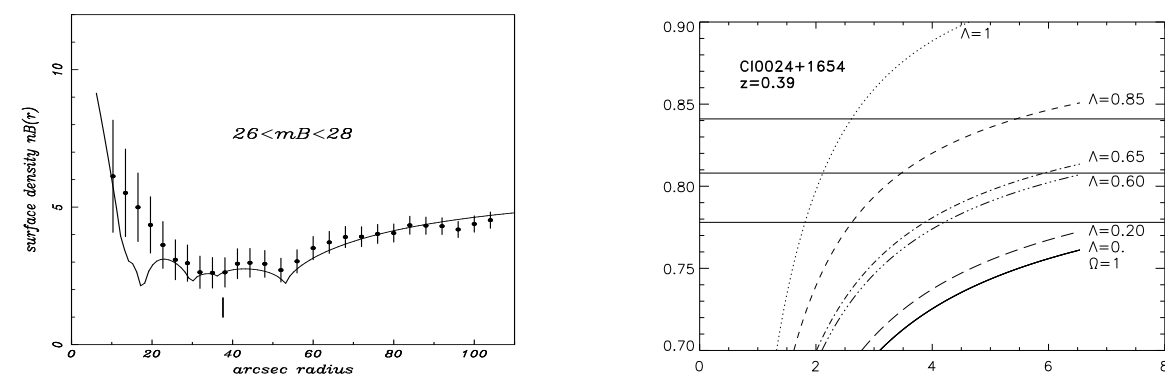

Fig. 4. A measurement of the cosmological constant from a depletion curve. The left panel shows the depletion curve measured in Cl0024+1654. By using the redshift corresponding to the two limits of the plateau observed on the curve, one can constrain the cosmological constant from the position of the second minimum. Whatever the redshift of the most distant sources visible on the images, we see that the angular position where the depletion curve raises again imposes that $\lambda>0.65$

with $\Omega_{\Lambda}>0.6$ (Kochanek 1995). Thus, if the conclusions of the magnification bias and of the QSO statistics are correct, few room is left for a possible value of the cosmological constant.

The Fort et al. (1996a) preliminary result is still questionable but at least it demonstrates that the magnification bias is worth to be explored further with many clusters, both to study the distribution and evolution of faint distant galaxies and to provide cosmological tests in clusters of galaxies with large multiple arcs. A reasonnable sample of 20 rich lensing clusters with simple geometry observed with FORS on the VLT would provide very deep exposures (to $B \approx 28-29$ ) rapidly with a very low flat field residuals which is crucial for detection in the noise. It will be a challenging programme with FORS on the VLT.

\section{Conclusion}

It is now established from the weak lensing analyses we have described here as well as from the HDF data and recent deep spectroscopic surveys (this conference) that it is possible to detect and study the population of galaxies at redshift beyond $z=4$. Most of them, despite probably strong star forming activities, will be close to or within the sky background noise. It is already demonstrated with 4 meter class telescopes that their detectability is not a big challenge for VLTs during period of excellent seeing. Their observations on a regular basis is a tremendous chance for weak lensing studies and their large cohort of exciting 
applications. However, for the measurement of very faint shear $(\approx 1 \%)$, it is crucial to control the stability of the image quality to a level which was never thought of before. A dedicated and a large comprehensive effort must be done in this domain by instrument builders and observers in order to reach the ultimate imaging capabilities of the VLTs.

\section{Acknowledgments}

We thank J.-C. Cuillandre, R. Pelló, P. Schneider, C. and S. Seitz, and L. Van Waerbeke for stimulating discussions about lensing and prospective aspects. J. Bézecourt kindly provided the spectra shown in this review.

\section{References}

Bézecourt, J., Soucail, G. 1996, SISSA preprint astro-ph/96006064.

Bonnet, H., Mellier, Y., Fort, B. 1994, ApJ 427, L83.

Bonnet, H., Mellier, Y. 1995, A\&A 303, 331.

Broadhurst, T., Taylor, A.N., Peacock, J. 1995, ApJ 438, 49.

Broadhurst, T. 1995, SISSA preprint astro-ph/9511150.

Cuillandre, J.-C., Fort, B., Picat, J.-P., Soucail, G., Altieri, B., Beigbeder, F., Dupin, J.-P., Pourthié, T., Ratier, G. 1994, A\&A 281, 603.

Ebbels, T. M. D., Le Borgne, J.-F., Pelló, R., Ellis, R. S., Kneib, J.-P., Smail, I., Sanahuja, B. 1996, SISSA astro-ph/9606015.

Fort, B., Mellier, Y. 1994, A\&A Review 5, 239, 292.

Fort, B., Mellier, Y., Dantel-Fort, M. 1996a, SISSA preprint astro-ph/9606039.

Fort, B., Mellier, Y., Dantel-Fort, M., Bonnet, H., Kneib, J.-P. 1996b, A\&A 310, 705.

Kneib, J.-P., Mathez, G., Fort, B., Mellier, Y., Soucail, G., Longaretti, P.-Y. 1994, A\&A 286, 701.

Kneib, J.-P., Ellis, R. S., Smail, I., Couch, W. J., Sharples, R. M. 1996, SISSA preprint astro-ph/9511015.

Kochanek, C. S. 1995, SISSA preprint astro-ph/9510077.

Mellier, Y., Van Waerbeke, L., Bernardeau, F., Fort, B. 1996, Proceedings of the VIIIth

Rencontres de Blois "Neutrinos, Dark Matter and the Universe". Blois, France 1996.

Narayan, R., Bartelmann, M. 1996, SISSA preprint astro-ph/9606001

Shanks, T. 1996, Proceedings of the $37^{\text {th }}$ Herstmonceux Conference "HST and the High Redshift Universe". Cambridge 1996. N. Tanvir, A. Aragón-Salamanca, J. V. Wall eds.

Smail, I., Hogg, D., Yan, L., Cohen, J. G. 1995, ApJ 449, L105.

Tyson, A. J. 1988, AJ 96, 1.

Tyson, J. A., Valdes, F., Wenk, R. 1990, ApJ 349, L1.

Van Waerbeke, L., Mellier, Y., Schneider, P., Fort, B., Mathez, G. 1996, A\&A in press. SISSA preprint astro-ph/9604137.

Van Waerbeke, L., Mellier, Y. 1996, Proceedings of the XXXIst Rencontres de Moriond "Dark Matter in Cosmology. Quantum Measurement, Experimental Gravitation". Les Arcs, France 1996. SISSA preprint astro-ph/9606100. 